\title{
Differential effects on sympathetic nerve activities elicited by activation of neurons in the pressor areas of dorsal and rostral ventrolateral medulla in cats
}

\author{
C.K. Su ${ }^{a}$, C.T. Yen ${ }^{b}$, J.C. Hwang ${ }^{c}$, C.J. Tseng ${ }^{d}$, J.S. Kuo ${ }^{\text {e }}$ and C.Y. Chai ${ }^{a}$ \\ ${ }^{a}$ Institute of Biomedical Sciences, Academia Sinica, ${ }^{b}$ Department of Zoology, National Taiwan Unicersity, ${ }^{c}$ Department of Biology, \\ National Taiwan Normal Unicersity, ${ }^{d}$ Department of Pharmacology, National Defense Medical Center and ${ }^{e}$ Department of Medical \\ Research, Taichung Veterans General Hospital, Taipei, Taiwan
}

(Received 17 October 1991)

(Revision received 14 May 1992)

(Accepted 5 June 1992)

Key words: Medullary pressor areas; Dorsal medulla; Ventrolateral medulla; Parvocellular reticular nucleus; Vertebral nerve; Renal nerve; Sympathetic nerve activity

\begin{abstract}
Changes of the nerve activity of the sympathetic renal and vertebral nerves were elicited by microinjection of sodium glutamate $(50 \mathrm{nmol} / 100 \mathrm{nl})$ into the pressor areas of the dorsal (DM) and rostral ventrolateral medulla (RVLM) in cats under urethane-chloralose anesthesia. Animals were bilaterally vagotomized, artificially ventilated, and paralyzed with gallamine triethiodide. The vertebral nerve activity always increased when pressor responses were induced by DM or RVLM stimulation. However, the effects of medullary stimulation on the renal nerve activity were variable. Three types of renal nerve responses concomitant with the pressor responses were observed in either baroreceptor-intact or baroreceptor-denervated cats. They were: (1) augmentation (type I); (2) attenuation (type II); and (3) insignificant change (type III). Type I responses were often elicited by RVLM stimulation whereas type II responses were often elicited by DM stimulation. Findings suggested that neurons integrating these sympathetic nerve activities were not equally distributed in the pressor areas of DM and RVLM. This result supports the notion that neurons located in different pressor areas of the brainstem exert differential effects over different sympathetic nerve activities.
\end{abstract}

\section{Introduction}

The rostral ventrolateral medulla (RVLM), specifically the area of 'subretrofacial nucleus' have been studied extensively and well documented for supplying the basal sympathetic tone essential for the maintenance of systemic arterial pressure (SAP) $[6,8,12,17,29,35,37,46]$. Similar types of experiments have shown that the dorsal

Correspondence to: C.Y. Chai, Institute of Biomedical Sciences, Academia Sinica, Nankang 11529, Taipei, Taiwan. medulla (DM), covering the areas of parvocellular nucleus (PVC) and the nucleus tractus solitarius (NTS), are also important in the integration of vasomotor activity $[2,10,21,36,40,42,47,48]$. Our previous studies $[24,25,39]$, using excitatory amino acids as a tool, have demonstrated the existence of pressor neurons in DM, activation of which produces a prominent increase of SAP. Moreover, bilateral destruction of the pressor neurons in DM with kainic acid significantly decreased the resting SAP, suggesting a role of $D M$ in maintaining vasomotor tone [39]. Furthermore, in studying the generation of sympathetic rhythms, 
Barman and Gebber [2] reported that the basal activity of RVLM sympathoexcitatory neurons were derived from that of DM. Such results add support to the findings of current investigations regarding SAP integration in the medulla.

Recent evidence indicates that activation of pressor neurons in RVLM may affect vascular beds differentially. For instance, Lovick [28] observed that application of DL-homocysteic acid to RVLM produced vasoconstriction or vasodilation of the hindlimb accompanied by vasoconstriction of the renal and mesenteric arteries. The neurons responsible for the vasoconstriction of the hindlimb were distributed more in the rostral than in the caudal RVLM. Such a topographical organization is similar to the findings of Dampney and McAllen [13] in that sympathetic fibers innervating the skin and muscle of the hindlimb may not respond uniformly to RVLM stimulation. In their study, sympathetic fibers innervating the skin were activated by stimulation of the medial part of the subretrofacial region while those innervating the muscle were activated by stimulating the lateral part of the same region. As these differential responses result from activation of different neuronal populations in the central nervous system, it is reasonable to speculate that DM may also affect the sympathetic outflow in a manner different from that of the RVLM.

The present study was conducted to compare the influence of DM and RVLM on sympathetic nerve activity. Renal sympathetic nerve originating from the celiac ganglion and vertebral sympathetic nerve originating from the stellate ganglion were chosen as indices to evaluate the responses of different sympathetic nerves elicited by microinjection of glutamate (Glu) on the pressor areas of DM and RVLM. Results of the present study suggest that DM and RVLM exert differential effects over sympathetic nerve activities.

\section{Materials and Methods}

\section{General procedure}

Twenty-eight cats of either sex $(2.0-3.3 \mathrm{~kg})$ were anesthetized with urethane $(400 \mathrm{mg} / \mathrm{kg})$ and $\alpha$-chloralose ( $40 \mathrm{mg} / \mathrm{kg}$ ), and paralyzed with gallamine triethiodide $(2 \mathrm{mg} / \mathrm{kg} / 30 \mathrm{~min})$. This regimen of anesthetics produced a stable condition of anesthesia for $8 \mathrm{~h}$ at least, as indicated by a steady level of blood pressure and heart rate. The femoral artery and vein were cannulated for measurement of SAP and administration of drugs. as described previously [9]. The trachea was intubated and artificial ventilation was adjusted to maintain an end tidal $\mathrm{CO}$, level within $4-4.5 \%$. The rectal temperature was kept at $37 \pm 0.5{ }^{\circ} \mathrm{C}$ with a feedback-controlled thermal blanket. Resting mean systemic arterial pressure (MSAP) was maintained above $80 \mathrm{mmHg}$, if necessary, by intravenous (iv.) infusion of $5 \%$ dextran solution (10 $\mathrm{ml} / \mathrm{h}$ ).

The animal's head was fixed in a David Kopf stereotaxic apparatus and occipital craniotomy was performed. The pressor sites in DM and RVLM were approached stereotaxically using the following coordinates: $3-5 \mathrm{~mm}$ rostral to the obex. 2-4 $\mathrm{mm}$ lateral to the midline, and $1-7 \mathrm{~mm}$ ventral from the dorsal surface of medulla. A 30-G stainless steel insulated needle, except its tip, was used as a monopolar electrode to explore the pressor sites of DM and RVLM, first by electrical stimulation $(100 \mu \mathrm{A}, 0.5 \mathrm{~ms}, 80 \mathrm{~Hz})$ then followed by chemical stimulation (sodium

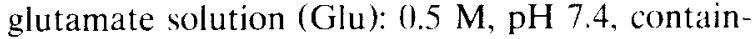
ing $1 \%$ Fast green in artificial CSF) $[9,11]$. By manually pushing the Hamilton syringe $(10 \mu \mathrm{l})$. which was connected to the electrode-tubing via a piece of PE-tube, the Glu solution was injected slowly $(100 \mathrm{nl} / 10-20 \mathrm{~s})$ to avoid volume effects. Histological verification showed that such an injection produced a stain of $300-750 \mu \mathrm{m}$ in diameter. Successive injections of Glu were made after an interval of at least $15 \mathrm{~min}$. In this way the effects were reproducible even when Glu was repeatedly applied to the same site two or three times. No more than 10 injections were made in an experiment. Control injection of the vehicle, the artificial CSF, did not produce any discernible effects.

All observations were made after bilateral vagotomy at the cervical level. Among the 28 cats, 17 cats were further subjected to baroreceptor denervation by sectioning both carotid sinus and aortic depressor nerves. Complete denervation 
was verified by the loss of reflex sympathoinhibition and bradycardia during SAP increase by phenylephrine (5 $\mu \mathrm{g} / \mathrm{kg}$, i.v.).

\section{Neural recordings}

The left renal or the left vertebral sympathetic nerves were approached dorsolaterally through incisions made in the abdominal or thoracic wall. Under an operating microscope, the nerves were dissected free from the surrounding connective tissues, desheathed, and cut at the distal end. A conventional electrophysiological setup and a bipolar platinum electrode were used to record and amplify the efferent whole-bundle nerve activities (bandpass: $10-3 \mathrm{k} \mathrm{Hz}$ ). The signals were monitored with an oscilloscope (Tektronix 5113) and stored on a tape recorder (Neuro Data DR-
886) for later analysis. Action potentials were rectified, and integrated by an integrator (Gould 13-4615-70) with a reset time of 1 or $5 \mathrm{~s}$. To acquire the actual neural activity (an example is shown in Fig. 1), the noise level of the recorded nerve contained in the integrated neurogram was measured by integrating the null nerve activity at $10 \mathrm{~min}$ after the animal was killed, and the noise value subtracted to calculate the actual nerve activity [49].

\section{Histology}

At the end of each experiment, the animal was sacrificed by an injection of a bolus of saturated $\mathrm{KCl}$. The brain was removed and fixed in $10 \%$ formalin. Frozen sections of the brain, $50 \mu \mathrm{m}$ thickness, were cut with a cryostat microtome and

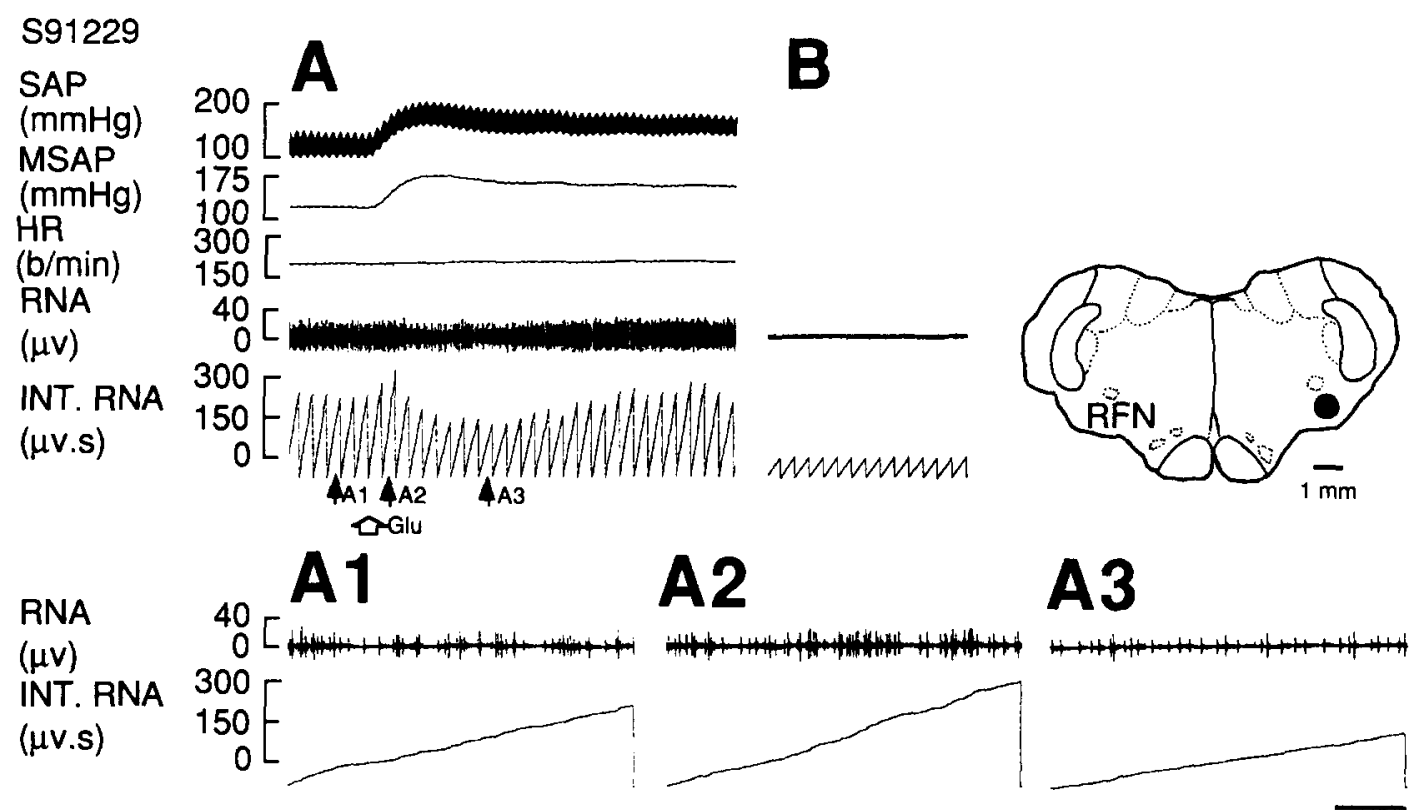

Fig. 1. An example showing the responses elicited by microinjection of Glu ( $100 \mathrm{nl}$ ) into RVLM in a cat with intact baroreceptors (S91229). A. A prolonged increase of SAP occurred concomitantly with the transient increase of RNA (excitatory phase) followed by prolonged inhibition of the nerve activity (inhibitory phase). A1. Fast tracings show the nerve activity before Glu injection. A2. Fast tracings show the nerve activity at the period of maximal excitation. A3. Fast tracings show the nerve activity in the inhibitory phase. B. The null nerve activity (background noise) at $10 \mathrm{~min}$ after the animal was killed. Histological verification of the injection site is indicated by the solid circle as shown in the brain drawing. The nerve activity was integrated with a reset time of 5 s. Horizontal calibrator represents $25 \mathrm{~s}$ in panels $\mathbf{A}$ and $\mathbf{B}$ or $1 \mathrm{~s}$ in panels $\mathbf{A 1}, \mathbf{A} 2$ and $\mathbf{A 3}$. Abbreviations in this and the following figures are: SAP, systemic arterial pressure; MSAP, mean SAP; HR, heart rate; RNA, renal nerve activity; INT. RNA, integrated RNA; VNA, vertebral nerve activity; INT. VNA, integrated VNA. Abbreviations in the brain drawings: 12n, hypoglossal nerve; 5SP, spinal trigeminal nucleus; ION, inferior olivary nucleus; RFN, retrofacial nucleus; ts, tractus solitarius. 
stained with Cresyl violet. Chemical stimulation sites were reconstructed from sections containing the electrode tracks and marks of the Fast green.

\section{Data analysis}

Percent changes for SAP increase and for the perturbation of nerve activity in response to microinjection of Glu into the pressor site were calculated by dividing the value of maximum change with the control value. As illustrated in Fig. 1, the control value of nerve activity was acquired by averaging the integrated nerve activities from six consecutive 5-s recordings before stimulation. The largest value deviated from the control level within $30 \mathrm{~s}$ after stimulation was considered as the value of maximum change, which was positive during excitation and negative during inhibition of the nerve activity. Percent changes of responses were grouped and classified according to the preparation of animals, injection sites, and types of nerve responses (explained latter). Significant change of a data set from the control level and difference between the relative data sets were verified by Student's $t$-test. Differences between the chances in observation of a specific type of nerve responses elicited by DM or RVLM stimulation were compared by the chisquare test. Data were presented as mean \pm standard error mean. $P$ value less than 0.05 was considered to be statistically significant.

\section{Results}

In 11 baroreceptor-intact and 17 baroreceptordenervated cats, a total of 64 sites in DM and 60 sites in RVLM were identified as pressor sites where increase of SAP $(>10 \%)$ were elicited by microinjection of Glu. The effects of chemical stimulation of these sites on SAP and sympathetic nerve activities are summarized in Table I. In the current preparations, Glu produced no significant effect on the heart rate in almost all the stimulations (an example is shown in Fig. 1).

TABLE I

Responses of systemic arterial pressure and sympathetic nerle acticities produced by microinjection of glutamate into the pressor areas of dorsal (DM) or rostral centrolateral medulla $(R V L M)$ in baroreceptor-intact or baroreceptor-denertated cats

\begin{tabular}{|c|c|c|c|c|c|c|}
\hline & \multicolumn{3}{|l|}{$\mathrm{DM}$} & \multicolumn{3}{|l|}{ RVLM } \\
\hline & Type I & Type II & Type III & Type 1 & Type II & Type III \\
\hline \multicolumn{7}{|c|}{ A. Renal nerce acticities ( $R N A)$} \\
\hline \multicolumn{7}{|l|}{ Intact cats } \\
\hline $\operatorname{SAP}(\mathscr{C})$ & $17 \pm 3 *$ & $29 \pm 5^{*}$ & $22 \pm 5 *$ & $34 \pm 5 *$ & $33 \pm 12$ & $33+9 *$ \\
\hline RNA $(\%)$ & $25 \pm 4^{*}$ & $-46 \pm 9 *$ & 0 & $34 \pm 5 *$ & $-47 \pm 18$ & 0 \\
\hline$n$ & 8 & 10 & 13 & 18 & 3 & 4 \\
\hline \multicolumn{7}{|c|}{ Baroreceptor-deneriated cats } \\
\hline $\operatorname{SAP}(\%)$ & $47 \pm 9 *$ & $36 \pm 12 *$ & $30 \pm 5 *$ & $37 \pm 6 *$ & $31 \pm 9 *$ & $25 \pm 9$ \\
\hline RNA (\%) & $30 \pm 8^{*}$ & $-40 \pm 6 *$ & 0 & $49 \pm 12 *$ & $-22 \pm 3 *$ & 0 \\
\hline$n$ & 11 & 8 & 5 & 22 & 3 & 6 \\
\hline \multicolumn{7}{|c|}{$\begin{array}{l}\text { B. Vertebral nerce acticities (VNA) } \\
\text { Intact cats }\end{array}$} \\
\hline SAP $(\%)$ & $34 \pm 14$ & - & - & $30 \pm 11$ & - & - \\
\hline VNA $(\%)$ & $46 \pm 10^{*}$ & - & - & $18 \pm 8$ & - & - \\
\hline$n$ & 5 & 0 & 0 & 3 & 0 & 0 \\
\hline \multicolumn{7}{|c|}{ Baroreceptor-denertated cats } \\
\hline $\operatorname{SAP}(\%)$ & $30 \pm 6^{*}$ & - & - & $25 \pm 3 *$ & - & - \\
\hline $\operatorname{VNA}(\%)$ & $101 \pm 22 *$ & - & - & $51 \pm 8 *$ & - & - \\
\hline$n$ & 14 & 0 & 0 & 15 & 0 & 0 \\
\hline
\end{tabular}

NA $(\%)$ : percentage change of nerve activities. SAP $(\%)$ : percentage change of systemic arterial pressure. All values are mean \pm S.E.M. $n=$ number of experiments. ${ }^{*}$ : changes statistically significani from the control value, $P<0.05$, calculated by Student's $t$-test. 
Only in three DM sites and four RVLM sites did the change of heart rate exceed $10 \%$, but the change was still minimal (less than $15 \%$ ). Therefore, the effects of stimulation on the heart rate were not analyzed further.

Changes in sympathetic nerve activity elicited by activation of the pressor areas of DM or RVLM

During activation of the pressor sites, the direction, magnitude and time course of sympathetic nerve responses varied extensively. The vertebral nerve activity (VNA) was consistently increased during DM or RVLM stimulation along with the SAP rise. In contrast, changes in the renal nerve activity (RNA) were not consistent in spite of the fact that SAP rise was always elicited by the stimulation. As shown in Fig. 2, three types of renal nerve responses were observed; (1) augmentation (type I); (2) attenuation (type II); and (3) insignificant change (less than $10 \%$ of the control value) of the nerve activity (type III).

The type I renal nerve responses observed in cats with intact baroreceptors could be further divided into an excitatory and an inhibitory phase
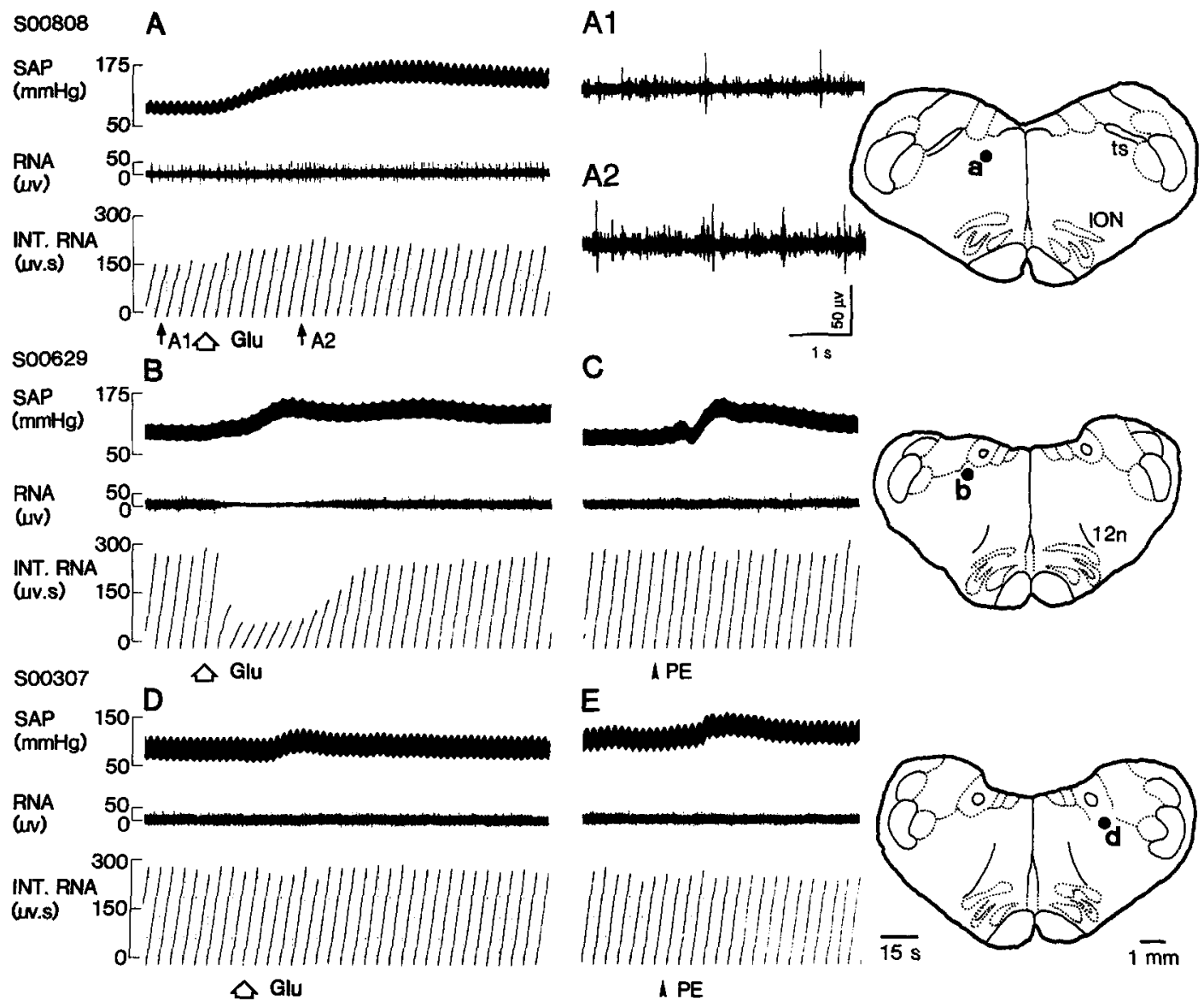

C

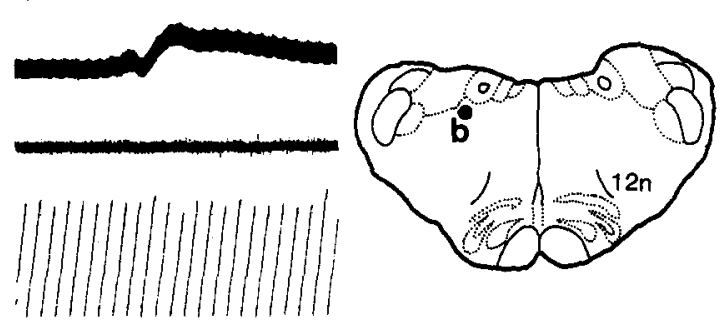

IPE

E

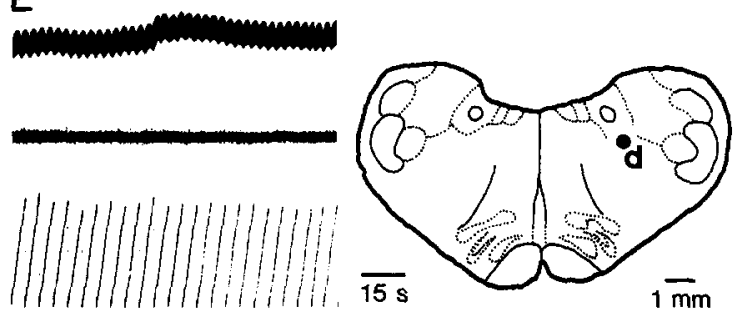

$\triangle \mathrm{PE}$

Fig. 2. Three types of RNA produced by microinjection of Glu ( $100 \mathrm{nl}$ ) into the pressor sites of DM (dots in the brain drawing) in three baroreceptor-denervated cats (S00808, S00629, S00307). A. Type I response: the nerve activity increased parallel with the pressor response. Fast tracings at top center illustrate a small segment of the neurogram before (A1) and after (A2) Glu stimulation. B. Type II response: the inhibition of nerve activity and SAP rise occurred simultaneously. Such inhibition was not secondary to baroreceptor reflex as phenylephrine (PE, $20 \mu \mathrm{g} / \mathrm{kg}$, i.v.) produced a greater SAP rise but no RNA inhibition (C). D. Type III response: microinjection of Glu produced an increase of the SAP but little change of the nerve activity. Similar effects were observed after a small dose, $(5 \mu \mathrm{g} / \mathrm{kg}$, i.v.), of phenylephrine (E). 
(Fig. 1A). Within a few seconds after the injection, RNA exhibited a rapid initial increase, reached their peak values earlier than the plateau of SAP rise and then was followed by an immediate decay. Following the excitatory phase, a prominent inhibition of the nerve activity, the inhibitory phase, was always observed. The duration of the excitatory phase was short $(5-30 \mathrm{~s})$, in contrast to the prolonged period of inhibition $(1-10 \mathrm{~min})$. The type I renal nerve responses in baroreceptor-denervated cats were different from that in baroreceptor-intact cats. In the baroreceptor-denervated cats, excitation of the nerve activity was stronger and the time course of the change of nerve activity was concordant with the change of SAP (Fig. 2A).

The type II renal nerve responses were characterized by an inhibition without any preceding excitation of sympathetic nerve activity during the SAP increase. These responses were observed in both baroreceptor-intact (Fig. 3) and -denervated cats (Fig. 2B). In animals with intact baroreceptors, most stimulations produced inhibition of nerve activity simultaneously with the onset of pressor responses. In some stimulations, the nerve activity decreased earlier than the SAP rise (Fig. 3 ), suggesting the inhibition was a result of direct activation of the inhibitory component, but not secondary to SAP increase.

Type III renal nerve responses were characterized by an insignificant change of nerve activity in spite of a substantial increase of SAP (Fig. 2C).

\section{Comparison between DM and RVLM actication} Changes of RNA following DM or RVLM stimulation. Several differences were observed between the RNA responses produced by DM and RVLM stimulation (Table I). First, RVLM stimulation produced stronger augmentation of the RNA (Type I response) than DM stimulation did in both the baroreceptor-denervated animals $(49 \pm$ $12 \%$ in RVLM versus $30 \pm 8 \%$ in DM, $P<0.05)$ and the baroreceptor-intact animals $(34 \pm 5 \%$ in RVLM vs. $25 \pm 4 \%$ in DM, $P<0.025)$. It is noteworthy that in the baroreceptor-denervated cats, the SAP increase elicited by RVLM stimulation was even smaller than that of DM stimulation $(37 \pm 6 \%$ vs. $47 \pm 9 \%)$. Second, in baroreceptor-
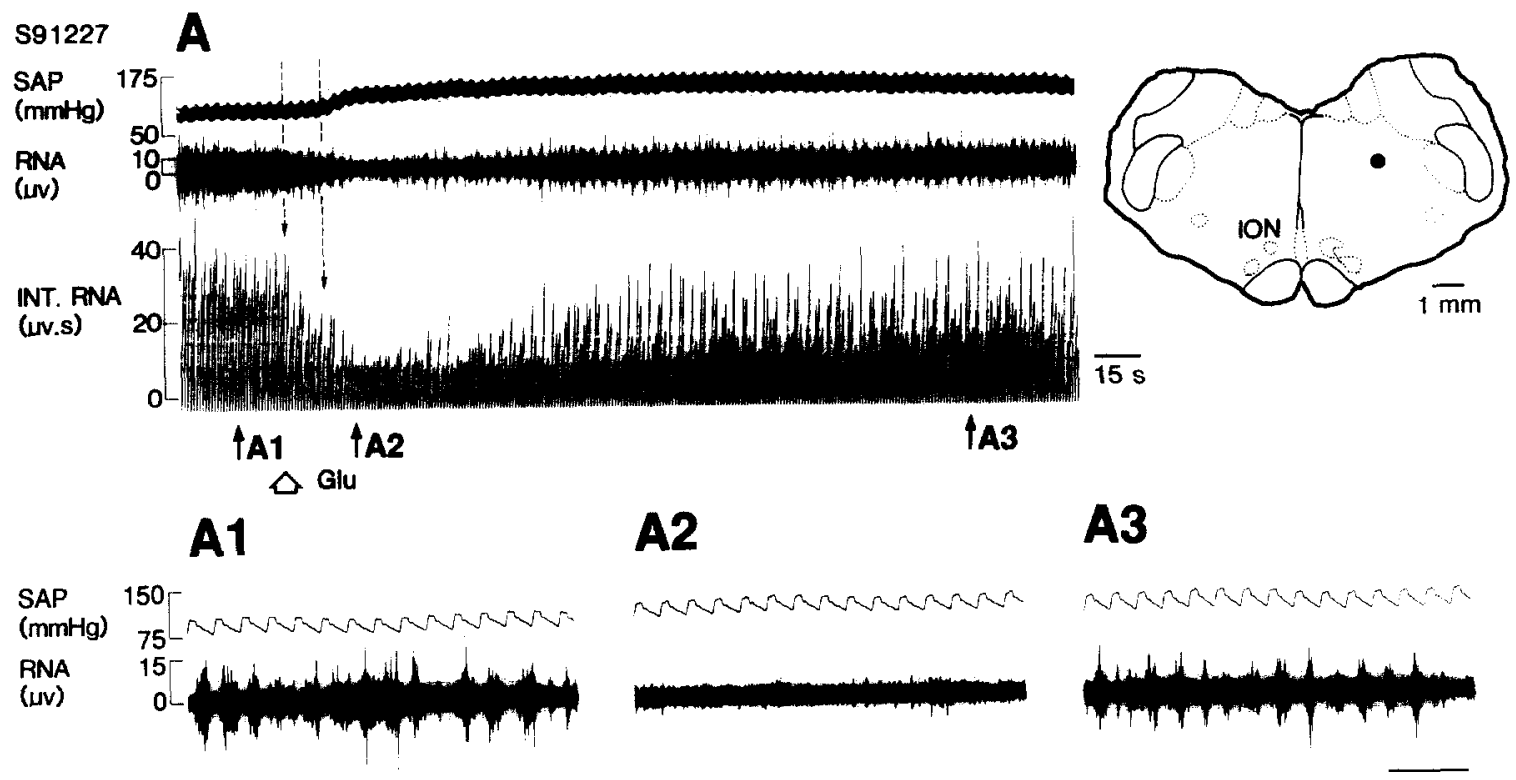

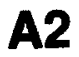

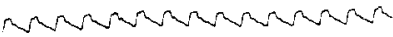

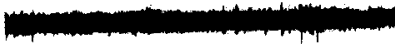

A3

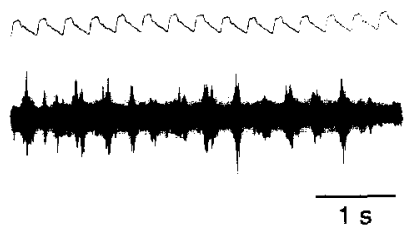

Fig. 3. An example showing that the decrease of RNA occurred earlier than the increase of SAP in a baroreceptor-intact cat (S91227). A the latency between the onset of RNA inhibition and SAP rise was $13 \mathrm{~s}$ (indicated by the dashed lines). Bottom panels showing the fast tracings of nerve activity before Glu injection $(100 \mathrm{nl})(\mathbf{A 1})$, showed the maximum inhibition of the nerve activity 23 $s$ after Glu (A2), and its recovery (A3). The nerve activity was integrated with a reset time of $1 \mathrm{~s}$. 
intact animals the frequency of occurrence of type I responses (Table IA) was significantly higher in RVLM stimulation $(18 / 25$ or $72 \%$ in RVLM vs. $8 / 31$ or $26 \%$ in DM, $P<0.025)$. Third, in baroreceptor-denervated animals the magnitude of RNA inhibition (type II responses in Table 1A) was more pronounced in DM stimulation $(-40 \pm 6 \%$ in DM vs. $-22 \pm 3 \%$ in RVLM,
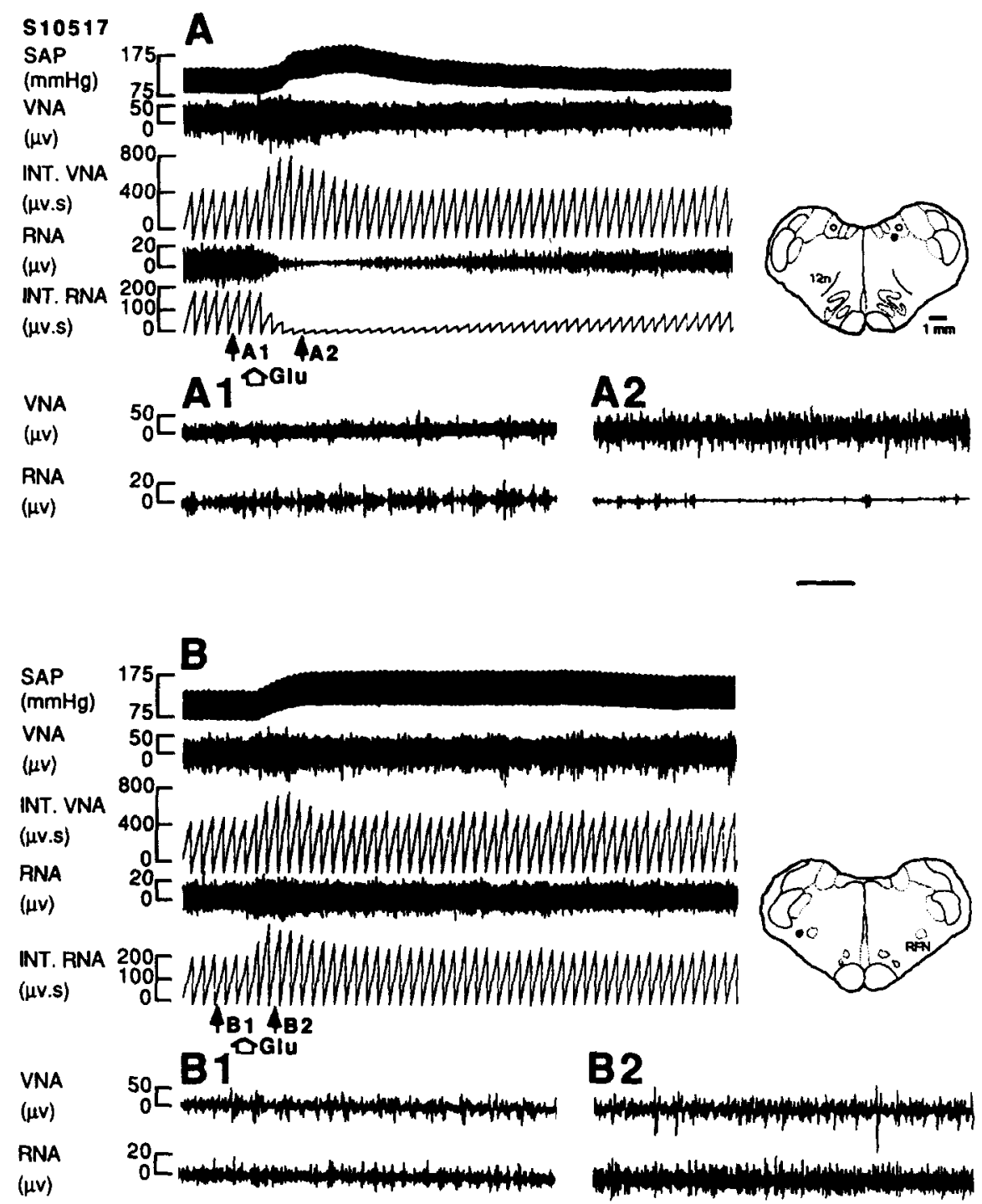

Fig. 4. Changes of the VNA and RNA elicited by microinjection of Glu into the pressor areas of DM and RVLM in a baroreceptor-denervated cat (S10517). A. A substantial SAP rise concomitant with increase of VNA but decrease of RNA was elicited by Glu $(100 \mathrm{nl})$ at DM. The change lasted for $50 \mathrm{~s}$ in VNA and up to 5 min in RNA. Al. The fast tracings show the nerve activities before Glu injection. A2. The fast tracings show the increase of VNA in contrast to the decrease of RNA after Glu injection. B. A prominent SAP rise concomitant with the increase of both VNA and RNA was elicited by a same dose of Glu at RVLM in the same animal. B1. The fast tracings show the nerve activities before Glu injection. B2. The fast tracings show the increase of both nerve activities after Glu injection. Note that the patterns of pressor response in A (decaying impetuously) and in B (decaying gradually) were different. Note also that the maximum increase of VNA over its control value was $86 \%$ in DM and $45 \%$ in RVLM. Horizontal calibrator represents $25 \mathrm{~s}$ in panels $\mathbf{A}$ and $\mathbf{B}$ or $1 \mathrm{~s}$ in panels $\mathbf{A 1}, \mathbf{A 2}, \mathbf{B 1}$ and $\mathbf{B 2}$ 
$P<0.005$ ). These findings suggest that the RVLM is more powerful in RNA excitation while the DM is more effective in RNA inhibition. Change of VNA following DM or RVLM stimulation. Difference between the responses of VNA by DM and RVLM stimulation was also observed (Fig. 4, Table IB). The excitatory effect of VNA consequent to DM stimulation was more pronounced in either baroreceptor-intact $(46 \pm 10 \%$ in DM vs. $18 \pm 8 \%$ in RVLM, $P<0.05)$ or baroreceptor-denervated cats $(101 \pm 22 \%$ in DM vs. $51 \pm 8 \%$ in RVLM, $P<0.001$ ), suggesting that the DM is more powerful to evoke VNA excitation.

In two baroreceptor-intact and four baroreceptor-denervated cats, RNA and VNA were simultaneously recorded in order to further cross-correlate their changes in response to DM or RVLM stimulation. As shown in Fig. 4, VNA was consistently increased either on DM or RVLM stimula-
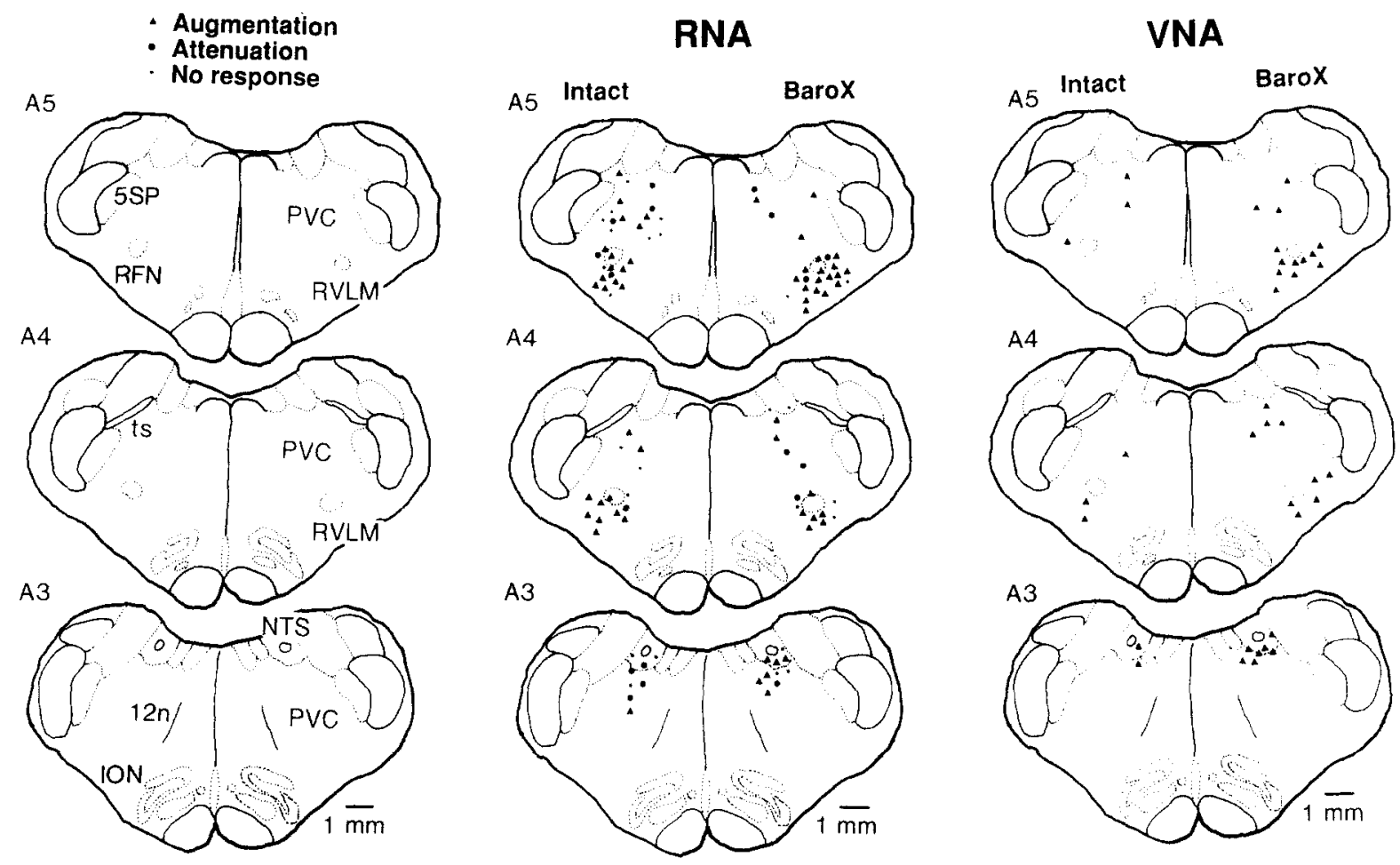

Fig. 5. Distribution of medullary sites where microinjection of Glu produced pressor responses with different types of nerve activity changes. Triangles, circles and small stars in the brain drawings represent the sites where type I (augmentation), type II (attenuation) and type III (change less than $10 \%$ of the control) nerve responses were elicited, respectively. The microinjection sites, either in the left or right side of the brain, are reconstructed and pooled in the same side. Left side represents the observations made in baroreceptor-intact cats, while in the right side baroreceptor-denervated cats observations are shown. In the left halves of the left drawings, the $12 n$ (hypoglossal nerve), the 5SP (spinal trigeminal nucleus), the ION (inferior olivary nucleus), the RFN (retrofacial nucleus) and the ts (tractus solitarius) are used as histological landmarks. In the right halves of these drawings, the approximate localization of the NTS (nucleus tractus solitarius), the PVC (parvocellular nucleus) and the RVLM (rostral ventrolateral medulla) are indicated. $\mathbf{A 5}, \mathbf{A 4}, \mathbf{A 3}=5,4,3 \mathrm{~mm}$ rostrol to the obex, respectively. In the middle drawings, RNA, in either baroreceptor-intact or baroreceptor-denervated (BaroX) cats, most of the type I renal nerve responses were aggregated in the region of RFN and scattered in the region of PVC at $\mathbf{A 4 - A 5}$. Points giving type II and III responses were found mainly on the level of A3. Some were scattered at levels of A4-A5 close to the area where type I responses were elicited. In VNA (the right drawings), only augmentation of VNA was elicited at the pressor sites in the regions of RFN, PVC and NTS. 
tion, whereas RNA was inhibited on DM stimulation.

Histological distribution of the pressor sites in correlation with different types of nerve responses

Distribution of reactive sites where microinjection of Glu produced pressor responses and three different types of nerve activity is shown in Fig. 5. The pressor sites in RVLM were confined to the vicinity of the retrofacial nucleus (RFN), whereas the pressor sites in DM were located around the areas of NTS and PVC.

For RNA, most of the type I responses were elicited on RVLM and PVC stimulations at the level of 4 (A4) to $5 \mathrm{~mm}$ (A5) rostral to the obex. A higher proportion of type II and III responses were produced on stimulation around NTS at the level of $3 \mathrm{~mm}$ (A3) rostral to the obex in cats with intact baroreceptors, but not with baroreceptordenervation. This suggests that a portion of the type II and III responses may be a result of baroreceptor activation secondary to SAP rise.

In contrast, only type I VNA responses were observed on stimulations of NTS, PVC and RVLM regardless of the integrity of baroreceptor reflex.

Comparison of effects elicited by electrical and Glu stimulation of DM

Electrical or Glu stimulation produced different patterns of sympathetic responses. As shown

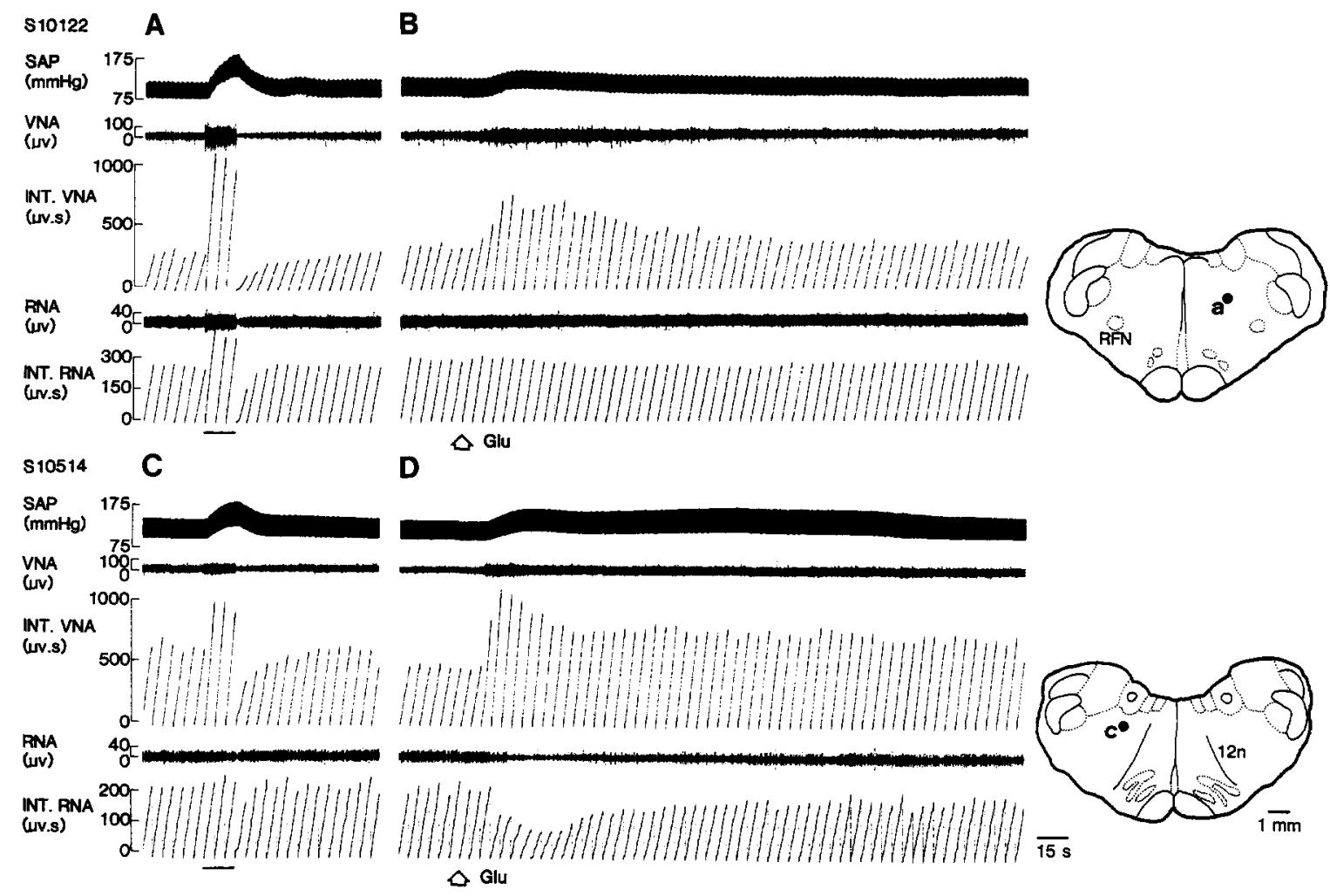

Fig. 6. Effects of electrical and Glu stimulations of DM on the vertebral and renal nerve responses in two baroreceptor-denervated cats (S10122, S10514). A. Both VNA and RNA were excited during electrical stimulation of the pressor point 'a' in PVC. B. Only a prominent increase of VNA concomitant with insignificant increase of RNA was elicited by microinjection of Glu (100 $\mathrm{nl}$ ) into the same point ' $a$ '. C. A prominent increase of VNA concomitant with a slight increase of RNA was elicited by electrical stimulation of a pressor point ' $c$ '. D. Only the VNA was excited while the RNA was inhibited after microinjection of Glu (100 $\mathrm{nl}$ ) into the same point ' $c$ '. Note that electrical and Glu stimulations produced similar responses in vertebral nerve but not in renal nerve. 
in Fig. 6, the effects elicited by electrical and Glu stimulations of DM were similar in VNA, running in a similar direction. The effects on RNA, however, were different. Electrical stimulation produced excitation, while Glu produced inhibition. Because Glu excites cell bodies and dendrites but spares the passing axons [18] while electrical current excites all of them, the differences between Glu and electrical stimulations may reflect the proportion of cells bodies and passing axons. It appears very likely that in DM the following components exist: (a) passing axons that may increase RNA upon activation; (b) neurons that inhibit RNA; and (c) neurons that excite RNA. Therefore, during electrical stimulation, the excitatory effects of passing axons and excitatory neurons (Fig. 6A-B) may mask the inhibitory effects resulting from activation of the inhibitory neurons (Fig. 6C-D). This also speaks for the advantage of using chemical instead of electrical stimulation.

\section{Discussion}

The major findings of the present study show that both RVLM and DM exert a differential effect over the vertebral and renal sympathetic nerves and that the patterns of effect differ. Activation of neurons in the pressor regions of RVLM and DM excited VNA but either excited, inhibited or had no effect on RNA. Neurons in DM had stronger excitatory influence over the vertebral sympathetic nerve, whereas those in RVLM excited the renal sympathetic nerve more.

The renal nerve is one of the postganglionic nerves originating from the celiac ganglion $[23,38]$ specifically for innervation of the kidney, where $1 / 5$ to $1 / 4$ blood flow of the cardiac output is regulated. The renal sympathetic nerve may also participate in the regulation of glomerular filtration rate and renin secretion [45]. The vertebral nerve, also a sympathetic nerve, originates from the stellate ganglion and courses along the vertebral artery [31]. It may be involved in the control of the vascular beds of the cervico-thoracic portion. However, the functional details have yet to be explored [7].
In the present study, the pressor areas of RVLM and DM were defined functionally, i.e., locations where significant pressor responses were elicited by Glu. In this sense, our samples of the RVLM were taken around the retrofacial nucleus, a region caudal to the facial nucleus and mostly ventral and lateral to the nucleus ambiguus at the level 4 to $5 \mathrm{~mm}$ rostral to the obex of the cat. In recent years, numerous experiments have shown that this region is critical to vasomotor functions. In cats, the evidence includes the connection of RVLM with the baroreceptor relay station, the NTS [27]; the connection with the intermediolateral column (IMLC) of the spinal cord where sympathetic preganglionic neurons reside [1]. Single unit recording experiments revealed that some neurons in this region receive baroreceptor inputs and/or modulate sympathetic activities [3,4]. Moreover, direct activation of RVLM neurons with excitatory amino acids produces strong pressor effect, whereas localized bilateral lesion produced pronounced hypotension [14].

Our samples of stimulation sites in DM were taken in and around the region of the rostral NTS and the parvocellular part of the medullary reticular formation. NTS at this level is rostral to the baroreceptor afferents' relay station but may receive the secondary projections from the baroreceptor inputs (Fig. 5 in [27]). DM is another area in the medulla for cardiovascular integration $[9,10,24,25,39]$. This area contains a large population of neurons projecting to IMLC [1]. Single unit recording experiments also revealed that DM neurons receive baroreceptor input and modulate sympathetic functions $[4,15]$.

Here we used Glu for neuron activation in the pressor areas of DM or RVLM. Microinjection of chemicals into the brain tissue has its own limitation and problem of diffusion [26]. In this study, after $100 \mathrm{nl}$ Glu injection the resolution area indicated by the Fast green contained in the vehicle reached a radius of $300-750 \mu \mathrm{m}$ close to the theoretical calculation by Nicholson's equation [32] of a radius of $610 \mu \mathrm{m}$.

The response of RNA to microinjection of Glu to DM or RVLM was not consistent. As shown in Fig. 5 and Table I, in many brain stimulations the 
elicited pressor response was accompanied with direct inhibition of RNA (type II nerve response). This inhibition might not be related to baroreceptor reflex activation because the decrease of nerve activity occurred much earlier than the onset of SAP rise (Fig. 3). Similar RNA inhibition during pressor response was also observed in animals after baroreceptor denervation (Figs. 2B, 4A and $6 \mathrm{D})$. This suggests that microinjection of Glu into the pressor areas of DM or RVLM activates simultaneously two categories of neurons that excite or inhibit RNA. On the other hand, the same stimulation always increased VNA, a result of activating the neurons that excite the vertebral nerve. This suggests a heterogeneous organization of the sympatho-excitatory and -inhibitory neurons in the medullary pressor areas. Furthermore, the proportion of sympatho-excitatory and -inhibitory neurons activated determines the pattern of the pressor responses. As shown in Fig. 4, the pressor response decayed impetuously (more sympathoinhibitory effect) when VNA increased but RNA decreased (Fig. 4A). The pressor response decayed gradually (less sympathoinhibitory effect) when both VNA and RNA were augmented (Fig. 4B).

The sympathetic nerve responses differ from each other both quantitatively and qualitatively. Qualitative differences included: (A) three types of renal nerve responses against only type I vertebral nerve response were found in DM or RVLM stimulation. This suggests a wider distribution of neurons that excite vertebral nerve in the medulla; and (B) Type I renal nerve responses occurred more frequently with RVLM than DM stimulation (Table IA), suggesting that the ratio of renal-excitatory to renal-inhibitory neurons is higher in RVLM than in DM. Quantitative differences included: (A) when the effect of baroreceptor reflex was unmasked (Table IA), the inhibitory effect on RNA elicited from DM ( -40 $\pm 6 \%$ ) was more potent than that from RVLM $(-22 \pm 3 \%)$, suggesting either a much denser population of renal-inhibitory neurons in the pressor area of $\mathrm{DM}$, or that these inhibitory neurons have a wider connection in the central nervous system; and (B) the excitatory effect on VNA elicited from DM $(101 \pm 22 \%)$ was more potent than that from RVLM $(51 \pm 8 \%)$, suggesting a much more dense population or a wider connection of the vertebral-excitatory neurons in DM.

Findings of differential effects over the renal and vertebral sympathetic nerves by DM and VLM are consistent with many recent observations. For example, when supraspinal control is removed by high cervical cord transection, the renal activities drop to $3 \%$ of the control level, the cardiac and splanchnic activities to less than $50 \%$, and the mesenteric and splenic activities show no change $[30,44]$. In unanesthetized decerebrated cats electrical stimulation of the nucleus raphe obscurus excites muscular sympathetic nerve but inhibits renal nerve activities [16]. In anesthetized cats we have shown that the paramedian reticular nucleus exerts stronger inhibitory effects on the splanchnic nerve during asphyxia than on the cardiac sympathetic nerve [49]. In anesthetized rabbits, Okada and $\mathrm{Ni}$ nomiya [34] reported that a wide range of changes of the cardiac and-renal sympathetic activities could be produced by electrical stimulation of the hypothalamus. Besides, many authors reported that RVLM neurons exert differential control over many sympathetic nerves. These include greater excitatory influence over the renal than the splenic nerves [5,19]; greater tonic influence over the renal than the mesenteric nerves [48]. The data in the present study confirm the strong renal sympathetic effects exerted by the RVLM.

The functional significance of differential integration of the sympathetic nerves in the central nervous system should be considered along with the following experimental findings. First, visceral or somatic afferent-stimulation produced different patterns of sympathetic changes. For example, baroreceptor $[20,33]$ chemoreceptor [22] and somatovisceral receptors [41] activations have all been shown to activate an unequal proportion of different sympathetic nerves. Second, during different physiological and pathological states unique patterns of sympathetic activities are observed. Examples are: during desynchronized sleep, muscular sympathetic fibers are active while renal sympathetic fibers are quiescent [16]; during the initial stage of a massive hemorrhage the 
renal sympathetic activity is suppressed while the adrenal sympathetic activity is increased to nearly $70 \%$ [43]. It is most likely that different central regions are called upon through afferent mechanism under different functioning states in effecting a differential control of different sympathetic activities.

Whether neurons in RVLM or DM have indeed been activated during various afferent stimulation or changes of the internal or external environments of the body is a question rarely examined. Do neurons in the RVLM or the DM respond as a functional unit? What are the average firing rates of these functional units? And how do these natural occurring firing rates correspond to the firing rates elicited with direct localized activation? These questions have to be answered in the future.

In conclusion, the present study demonstrates another example of the differential effects of the sympathetic activities. How these potentially useful mechanisms integrate into the normal functions of the animal awaits further experimentation.

\section{Acknowledgements}

The authors wish to thank Ms. J.J. Pan and Mr. G.T. Chen for preparation of the manuscript and illustrations. Special thanks are due to Dr. S.H. Ngai, Professor Emeritus, College of Physicians and Surgeons, Columbia University for reading of the manuscript, and to Dr. C.W. Wu for his support and advice. The study was supported in part by grants from Foundation of Biomedical Sciences, and the National Science Council, Republic of China, NSC80-0412-B-00102.

\section{References}

1 Amendt, K., Czachurski, J., Dembowsky, K. and Seller, H., Bulbospinal projection to the intermediolateral cell column; a neuroanatomical study, J. Auton. Nerv. Syst., 1 (1979) 103-117.

2 Barman, S.M. and Gebber, G.L., Lateral tegmental field neurons of cat medulla: a source of basal activity of ventrolateral medullospinal sympathoexcitatory neurons, 1. Neurophysiol., 57 (1987) 1410-1424.

3 Barman, S.M. and Gebber, G.L.. Axonal projection pat terns of ventrolateral medullospinal sympathoexcitatory neurons, J. Neurophysiol., 53 (1985) 1551-1566.

4 Barman, S.M. and Gebber, G.L.. Brainstem neuronal types with activity related to sympathetic nerve discharge. Am. J. Physiol., 240 (1981) R335-R347.

5 Beluli, D.J. and Weaver, L.C.. Differential control of renal and splenic nerves without medullary topography, Am. J. Physiol., 260 (1991) H1072-H1079.

6 Blessing, W.W., Goodchild, A.K., Dampney, R.A.L. and Chalmers, J.P., Cell groups in the lower brain stem of the rabbit projecting to the spinal cord, with special reference to catecholamine-containing cells, Brain Res.. 221 (1981) $35-55$.

7 Bulygin, I.A., A consideration of the general principles of organization of sympathetic ganglia, J. Auton. Nerv. Syst., 8 (1983) 303-330.

8 Calaresu, F.R. and Yardley C.P., Medullary basal sympathetic tone, Annu. Rev. Physiol, 50 (1988) 511-524.

9 Chai, C.Y., Lin, R.H., Lin, A.M.Y., Pan, C.M., Lee, E.H.Y. and Kuo, J.S., Pressor responses from electrical or glutamate stimulation of the dorsal or ventrolateral medulla., Am. J. Physiol., 255 (1988) R709-R717.

10 Chai, C.Y. and Wang. S.C. Integration of sympathetic cardiovascular mechanisms in medulla oblongata of the cat, Am. J. Physiol., 215 (1968) 1310-1315.

11 Chiang, C.H. and Pappagianopoulos, P.. Central cardiorespiratory effects of glutamate in dogs, J. Appl. Physiol., 60 (1986) 2056-2062.

12 Ciriello, J., Caverson, M.M. and Polosa, C., Function of the ventrolateral medulla in the control of the circulation. Brain Res. Rev., 11 (1986) 359-391.

13 Dampney, R.A.L. and McAllen, R.M., Differential control of sympathetic fibers supplying hindlimb skin and muscle by subretrofacial neurons in the cat, J. Physiol. 395 (1988) 41-56.

14 Dampney, R.A.L. and Moon, E.A., Role of the ventrolateral medulla in vasomotor response to cerebral ischemia. Am. J. Physiol., 239 (1980) H349-H358.

15 Felder, R.B., Excitatory and inhibitory interactions among renal and cardiovascular afferent nerves in dorsomedial medulla, Am. J. Physiol., 250 (1986) R580-R588.

16 Futuro-Neto, H.A. and Coote, J.H., Changes in sympathetic activity to heart and blood vessels during desynchronized sleep, Brain Res., 252 (1982) 259-268.

17 Gatti, P.J. and Gillis, R.A., Interaction between pressor and depressor areas in cat ventrolateral medulla, Brain Res., 552 (1991) 153-158.

18 Goodchild, A.K., Dampney, R.A.L. and Bandler, R.A.. A method for evoking physiological responses by stimulation of cell bodies, but not axons of passage, within localized regions of the central nervous system, J. Neurosci. Methods, 6 (1982) $351-363$.

19 Hayes, K. and Weaver, L.C., Selective control of sympathetic pathways to the kidney, spleen and intestine by the 
ventrolateral medulla in rats, J. Physiol., 428 (1990) 371385.

20 Kendrick, E., Oberg, B. and Wennergren, G., Vasoconstrictor fibre discharge to skeletal muscle, kidney, intestine and skin at varying levels of arterial baroreceptor activity in the cat, Acta Physiol. Scand., 85 (1972) 464-476.

21 King, K.A. and Pang, C.C., Cardiovascular effects of injections of vasopressin into the nucleus tractus solitarius in conscious rats, Br. J. Pharmacol., 90 (1987) 531-536.

22 Kollai, M. and Koizumi, K., Differential responses in sympathetic outflow evoked by chemoreceptor activation, Brain Res., 138 (1977) 159-165.

23 Kuo, D.S., de Groat, W.C. and Nadelhaft, I., Origin of sympathetic efferent axons in the renal nerves of the cat, Neurosci. Lett., 29 (1982) 213-218.

24 Lin, R.H., Lin, A.M.Y., Su, C.K., Kuo, J.S. and Chai, C.Y., Presence of perikarya for vasopressor actions in both the dorsal and ventrolateral regions of medulla oblongata in swine, Neurosci. Res. Commun., 5 (1989) 125-133.

25 Lin, A.M.Y., Wang, Y., Kuo, J.S. and Chai, C.Y., Homocysteic acid elicits pressor responses form ventrolateral medulla and dorsomedial medulla, Brain Res. Bull., 22 (1988) 627-631.

26 Lipski, J., Bellingham, M.C., West, M.J. and Pilowsky, P., Limitation of the technique of pressure microinjection of excitatory amino acids for evoking responses from localized regions of the CNS, J. Neurosci. Methods, 26 (1988) 169-179.

27 Loewy, A.D. and Burton, H., Nuclei of the solitary tract: Efferent projections to the lower brain stem and spinal cord of the cat, J. Comp. Neurol., 181 (1978) 421-450.

28 Lovick, T.A., Differential control of cardiac and vasomotor activity by neurons in the paragigantocellularis in the cat, J. Physiol., 389 (1987) 23-35.

29 McAllen, R.M., Location of neurons with cardiovascular and respiratory function, at the ventral surface of the cat's medulla, Neurosci., 18 (1986) 43-49.

30 Meckler, R.L. and Weaver, L.C., Splenic, renal and cardiac nerves have unequal dependence upon tonic supraspinal inputs, Brain Res., 338 (1985) 123-135.

31 Nagashima C. and Kazuo, I., Electrical stimulation of the stellate ganglion and the vertebral nerve, J. Neurosurg., 36 (1972) 756-762.

32 Nicholson, C., Diffusion from an injected volume of a substance in brain tissue with arbitrary volume fraction and tortuosity, Brain Res., 333 (1985) 325-329.

33 Ninomiya, I., Nisimaru, N. and Irisawa, H., Sympathetic nerve activity to the spleen, kidney, and heart in response to baroreceptor input, Am. J. Physiol., 221 (1971) 13461351.

34 Okada, Y. and Ninomiya, I., Different cardiac and renal inhibitory and excitatory areas in rabbit hypothalamus, Am. J. Physiol., 244 (1983) H832-H838.

35 Pilowsky, P., West, M. and Chalmers, J., Renal sympa- thetic nerve responses to stimulation, inhibition and destruction of the ventrolateral medulla in the rabbit, Neurosci. Lett., 60 (1985) 51-55.

36 Raby, W.N. and Renaud, L.P., Dorsomedial medulla stimulation activates rat supraoptic oxytocin and vasopressin neurons through different pathways, J. Physiol. (Lond.), 417 (1989) 279-294.

37 Reis, D.J., Ross, C.A., Ruggiero, D.A., Granata, A.R. and Joh, T.H., Role of adrenaline neurons of ventrolateral medulla (the $\mathrm{C} 1$ group) in the tonic and phasic control of arterial pressure, Clin. Exp. Hyper, A6 (1984) 221-241.

38 Sripairojthikoon, W. and Wyss, J.M., Cells of origin of the sympathetic renal innervation in rat, Am. J. Physiol., 252 (1987) F957-F963.

39 Su, C.K., Lin, A.M.Y., Lin, R.H., Kuo, J.S. and Chai, C.Y., Contribution between dorsal and ventrolateral regions of medulla oblongata in vasomotor function of cats, Brain Res. Bull., 23 (1989) 447-456.

40 Talman, W.T. and Robertson, S.C., Glycine, like glutamate microinjected into the nucleus tractus solitarii of rat decreases arterial pressure and heart rate, Brain Res., 477 (1989) 7-13.

41 Toby, J.C. and Weaver, L.C., Pressoreceptor modulation of renal but not splenic sympathetic reflexes, Am. J. Physiol., 252 (1987) R26-R33.

42 Urbanski, R.W. and Sapru, H.N., Evidence for a sympathoexcitatory pathway from the nucleus tractus solitarii to the ventrolateral medullary pressor areas, J. Auton. Nerv. Syst., 23 (1988) 161-174.

43 Victor, R.G., Thoren, P., Morgan, D.A. and Mark, A.L., Differential control of adrenal and renal sympathetic nerve activity during hemorrhagic hypotension in rats, Circ. Res., 64 (1989) 686-694.

44 Weaver, L.C. and Stein R.D., Effects of spinal cord transection on sympathetic discharge in decerebrate-unanesthetized cats, Am. J. Physiol., 257 (1989) R1506R1511.

45 West, J.B., Edited Best and Taylor's Physiological Basis of Medical Practice, 11th Edn. pp. 461, Williams and Wilkins.

46 Willette, R.N., Punnen-Grandy, S., Krieger, A.J. and Sapru, H.N., Differential regulation of regional vascular resistance by the rostral and caudal ventrolateral medulla in the rat, J. Auton. Nerv. Syst., 18 (1987) 143-151.

47 Yardley, C.P., Andrade, J.M. and Weaver, L.C., Evaluation of cardiovascular control by neurons in the dorsal medulla of rats, J. Auton. Nerv. Syst., 29 (1989) 1-12.

48 Yardley, C.P., Stein, R.D. and Weaver, L.C., Tonic influences from the rostral medulla affect sympathetic nerves differentially, Am. J. Physiol., 256 (1989) R323-R331.

49 Yen, C.T., Lin, Y.F., Hwang, J.C., Su, C.K. and Chai, C.Y., Differential control of sympathetic nerve activities during combined asphyxia and paramedian reticular nucleus stimulation in cats, Neurosci. Res. Commun., 8 (1991) 95-102. 